\title{
Interference of Silver Nanoparticles with Tumor Necrosis Factor Action in Epithelial Cells
}

\author{
Grądzka Iwona, Sikorska Katarzyna, Brzóska Kamil \\ Institute of Nuclear Chemistry and Technology, Centre for Radiobiology and Biological Dosimetry \\ Dorodna 16 St. 03-195 Warsaw, Poland \\ i.gradzka@ichtj.waw.pl; k.sikorska@ichtj.waw.pl; k.brzoska@ichtj.waw.pl
}

\section{Extended Abstract}

Silver nanoparticles (AgNPs), due to their antibacterial properties are widely used as additives to textiles, cosmetics, food packaging, surgical instruments or wound dressings. Being present in so many consumer goods, AgNPs are able to penetrate the human body via multiple paths [1]. The formation of reactive oxygen species (ROS) is believed to be the main mechanism of AgNPs action in a cell. ROS can launch both pro-survival and death signaling pathways depending on the level of oxidative stress induced [2]. Recently, we showed that AgNPs are able to activate NF- $\kappa$ B signaling pathway and expression of genes related to inflammatory and stress response [3]. Similar program is activated by tumor necrosis factor (TNF) - a major pro-inflammatory cytokine [4].

Our objective was to assess the possible interference of AgNPs (20 nm, BSA coated) with cellular response to TNF in two human cell lines: A549 lung adenocarcinoma and HepG2 liver hepatocellular carcinoma. Both types of cells absorbed AgNPs added to the medium as shown cytometrically by using side-scattered light. During 24-hour incubation, the effect of TNF and AgNPs on growth retardation and the incidence of cell death was additive and HepG2 cells were more sensitive to the agents studied. Analysis of the cell cycle discovered G1 arrest after TNF and S and G2/M arrest under AgNPs influence in both cell lines, whereas the combined treatment resulted in G1 and S accumulation in A549 and G2/M accumulation in HepG2 cells. Over a longer incubation period (7-12 days), in the clonogenicity test, the effect of TNF and AgNPs on the cell survival was synergistic. The effect of the selected signaling pathways inhibitors was tested using neutral red viability 24-hour assay. Surprisingly, the use of IKK II and IKK VII - the inhibitors of I $\square$ B kinase on the prosurvival NF $\square$ B pathway - led to the increase of the cell viability in both cell lines treated with TNF, AgNPs or both agents simultaneously.

Apart from end cellular effects, the expression of genes involved in the anti-oxidative defense and inflammatory response was analyzed by using real-time PCR. It was shown that the expression of heme oxygenase 1 (HMOX1), the protein induced by oxidative stress, was greatly enhanced in TNF and AgNPs treated cells compared to that observed after TNF alone. Similarly, AgNPs augmented the expression of pro-inflammatory cytokines CSF3 and IL-10. On the contrary, the expression of toll-like receptors TLR3 and TLR7, important for virus pathogen recognition, was significantly hampered by the addition of AgNPs.

The presented results indicate that AgNPs change the final cellular result of TNF action and disrupt the cellular homeostasis, that can contribute to the development of malignancy or autoimmune diseases at the level of the organism. Therefore, an extended study is needed to provide more information about the nature and specificity of the functional interactions between TNF and AgNPs in cells.

This work was supported by the grant 2014/13/D/NZ7/00286 from National Science Centre, Poland.

\section{References}

[1] M. Kruszewski, K. Brzóska, G. Brunborg et al., "Toxicity of silver nanomaterial in higher eukaryotes", Adv. Mol. Toxicol., vol. 5, pp. 179-218, 2011.

[2] J. Rauch, W. Kolch, S. Laurent, M. Mahmoudi, "Big signals from small particles: regulation of cell signaling pathways by nanoparticles" Chem. Rev., vol. 113, no. 5, pp. 3391-3406, 2013. 
[3] T. M. Stępkowski, K. Brzóska, M. Kruszewski, "Silver nanoparticles induced changes in the expression of NF- $\kappa B$ related genes are cell type specific and related to the basal activity of NF-kB", Toxicol. In Vitro, vol. 28, no. 4, pp. 473-478, 2014.

[4] J. Neumeyer, A. Krippner-Heidenreich, "Tumor Necrosis Factor" in Encyclopedia of Molecular Pharmacology, S. Offermanns, W. Rosenthal, Eds. Berlin Heidelberg New York: Springer, 2008, pp. 1247-1251. 